\title{
Self-organized twinning of actuated particles for microfluidic pumping
}

Roy J. S. Derks, Arjan J. H. Frijns, Menno W. J. Prins, and Andreas H. Dietzel

Citation: Appl. Phys. Lett. 92, 024104 (2008);

View online: https://doi.org/10.1063/1.2834851

View Table of Contents: http://aip.scitation.org/toc/apl/92/2

Published by the American Institute of Physics

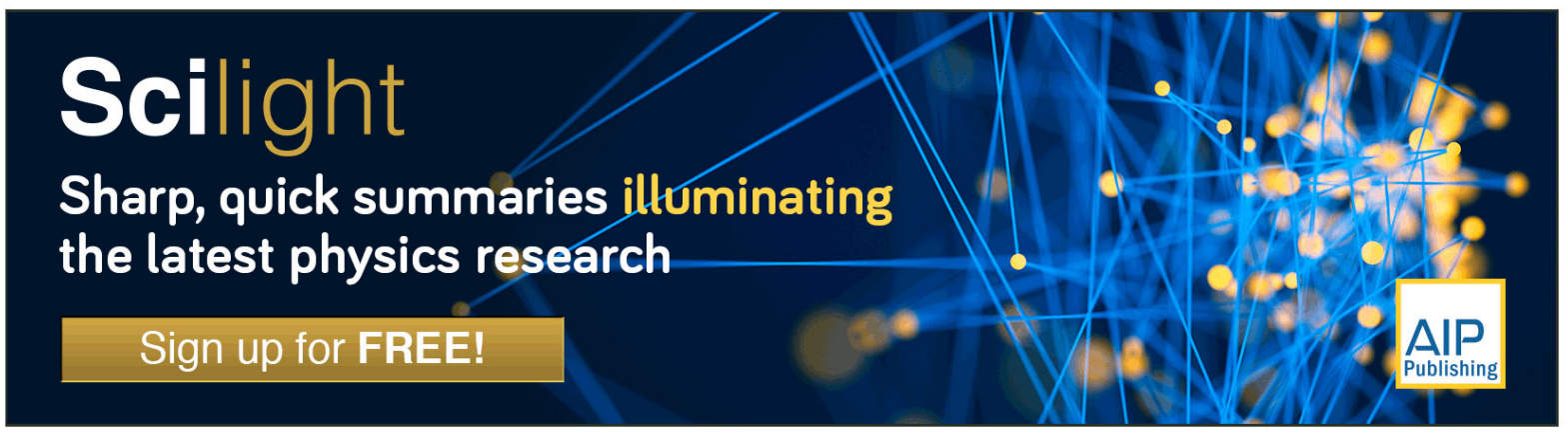




\title{
Self-organized twinning of actuated particles for microfluidic pumping
}

\author{
Roy J. S. Derks, ${ }^{1, a)}$ Arjan J. H. Frijns, ${ }^{1}$ Menno W. J. Prins, ${ }^{2}$ and Andreas H. Dietzel ${ }^{3}$ \\ ${ }^{1}$ Eindhoven University of Technology, Department of Mechanical Engineering, P.O. Box 513, \\ $5600 \mathrm{MB}$ Eindhoven, The Netherlands \\ ${ }^{2}$ Eindhoven University of Technology, Department of Applied Physics, P.O. Box 513, 5600 MB Eindhoven, \\ The Netherlands and Philips Research Europe, High Tech Campus 4, 5656 AE Eindhoven, \\ The Netherlands \\ ${ }^{3}$ Eindhoven University of Technology, Department of Mechanical Engineering, P.O. Box 513, \\ 5600 MB Eindhoven, The Netherlands and Holst Centre, High Tech Campus 31, P.O. Box 8550, \\ 5605 KN Eindhoven, The Netherlands
}

(Received 19 November 2007; accepted 19 December 2007; published online 14 January 2008)

\begin{abstract}
The motion of monodisperse particle ensembles in fluidic channels actuated by axial magnetic or gravitation forces is studied. Interactions between particles, fluid, and nearby walls induce unforeseen self-organization phenomena. Superparamagnetic microparticles aligned on a channel axis successively organize toward a stable polytwin system under constant force conditions. In the absence of repelling particle interactions, full contact twinning is observed for particles driven by gravity. The mechanisms of successive twinning and spacing regulation are explained by a one-dimensional model based on the axis flow profile. Related performance enhancements for particle based microfluidic pumping are discussed. (C) 2008 American Institute of Physics. [DOI: $10.1063 / 1.2834851]$
\end{abstract}

The engineering trends of miniaturization and integration promote lab-on-a-chip devices that employ small, multifunctional, and easy to control components. ${ }^{1}$ The use of superparamagnetic microparticles in biomedical diagnostic systems appears to be a very promising approach for sensitive sensing techniques, ${ }^{2}$ microscaled transport, ${ }^{3}$ and fluid mixing. ${ }^{4}$ Our research concerns the dynamics and interplay of these particles in confined geometries, aiming for microfluidic system applications. ${ }^{3-5}$ In this paper, magnetic and hydrodynamic interaction phenomena of monodisperse particles traveling along a channel axis are discussed; in particular, the observed self-organization to a polytwin system. These effects are studied to evaluate an application for particles as integrated fluid drivers in microfluidic systems.

Two types of experiments have been designed to investigate the motion and interplay of particle ensembles in a channel. Figure 1(a) shows an experimental platform developed for magnetic microparticle actuation. In a polycarbonate fluidic chip, a square microchannel (width/depth $100 \mu \mathrm{m}$; length $8 \mathrm{~mm}$ ) was created by excimer laser ablation. The channel in- and outlet are coupled by two backflow channels (total cross section of $2 \mathrm{~mm}^{2}$ ) to minimize external pressure drops. A glass slide was used to close the complete channel circuit. A yoke that is magnetized by a solenoid (not displayed) connects the adjoining pole tips. Their shape is designed to create a magnetic isodynamic force over their line of symmetry $\left(\nabla B^{2}\right.$ is constant, where $B$ is the magnetic field), which is aligned with the microchannel axis. ${ }^{3,6}$

The circuit was filled with a solution of superparamagnetic particles of $20 \mu \mathrm{m}$ in diameter (Spherotech, USA) that travel through the microchannel under the action of the applied magnetic field $\left(\nabla B^{2} \approx 0.2 \mathrm{~T}^{2} / \mathrm{m}\right.$, individual particle force $20 \mathrm{fN}$ ). The channel walls induce a hydrodynamic levitation to focus and line up the particles along the channel axis with an initially arbitrary interspacing. ${ }^{7}$ We noticed a

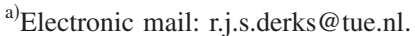

repetitive self-organization for the particles in this configuration. The two particles in front of the moving row reduce their interspacing, form a twin and travel away with an increased velocity, as shown in Fig. 1(b). The next heading
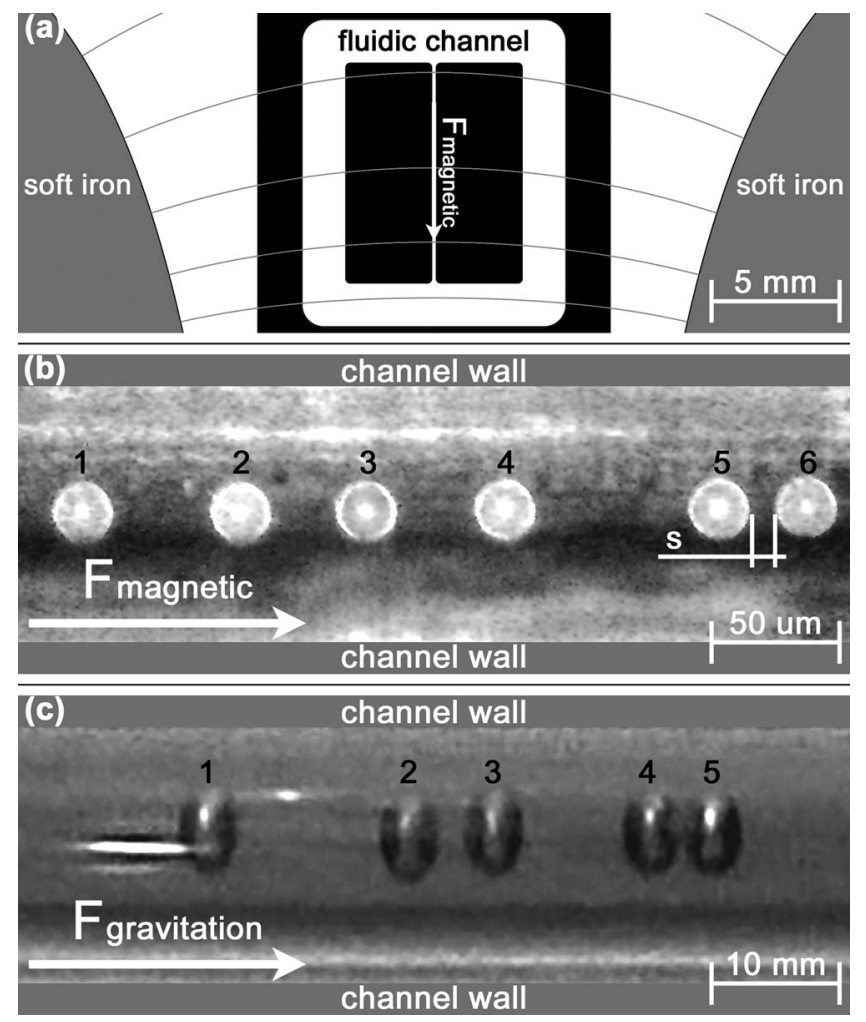

FIG. 1. (a) Sketch of the experimental platform with pole tips that create an isodynamic magnetic field over the microchannel. (b) Traveling superparamagnetic particles $(20 \mu \mathrm{m})$, focused on the axis of a microchannel by hydrodynamic wall interactions. A twin is formed at the front of the row and travels away, but keeps a small intraspacing $(s)$ caused by repelling magnetic forces. (c) Steel particles $(5 \mathrm{~mm})$ experiment where gravity provides a constant particle force. Only hydrodynamic particle interactions occur that allow twins to fully come in contact, as particles 4 and 5 show. Particles 3 and 4 are still at the onset of twinning. 
particles follow successively the same reorganization processes, through which the row shortens until a full polytwin system is formed. Remarkably, the twinned microparticles do not completely touch. In addition to the hydrodynamic interaction forces, nearby particles experience a repelling magnetic dipole force induced by the applied magnetic field perpendicular to the motion direction. These forces appear to equilibrate at an intraspacing $(s)$ in the order of a particle diameter.

A second setup has been built for gravitation driven studies. A plastic tube of $1 \mathrm{~m}$ in length and $15 \mathrm{~mm}$ in diameter was placed in vertical direction to use gravity for a constant particle force. It was closed at the bottom to reflect infinite channel length conditions. Stainless steel particles are used with a diameter of $5 \mathrm{~mm}$ and a density of $7860 \mathrm{~kg} / \mathrm{m}^{3}$. To meet the low Reynolds number regime $(\operatorname{Re} \ll 1)$ as in the magnetic particle experiment, the tube was filled with highly viscous oil (SAE 80W90, Comma, United Kingdom, density $887 \mathrm{~kg} / \mathrm{m}^{3}$, viscosity $200 \mathrm{mPa}$ s at $20^{\circ} \mathrm{C}$ ). In the gravitation experiments, the number of particles and their initial interspacing were varied, and all experiments confirmed the twinning effect, as shown in Fig. 1(c). Moreover, we observed fully developed twins with particles in complete contact. The gravitation driven particles are not subjected to (short range) repelling forces in contrast with the microparticles driven by magnetics, proving that successive twinning is induced by hydrodynamic interactions.

In order to explain the observed interaction phenomena we developed a one-dimensional (1D) model that only considers the channel axis flow profile, allowed by the systems axial symmetry. One or more spherical monodisperse particles with radius $(r)$ are positioned on the axis of an infinitely long channel with radius $(R)$ and are surrounded by a fluid with density $\left(\rho=10^{3} \mathrm{~kg} / \mathrm{m}^{3}\right)$ and viscosity $(\eta$ $=1 \mathrm{mPa}$ s). On every single particle an identical force $(F)$ is applied in axial direction. The model assumes a fully developed laminar flow $(\operatorname{Re} \ll 1)$ and particles that are large enough not be disturbed by Brownian motion (actuation energy dominates over thermal energy). In this regime, the model is fully scalable by the ratio of channel to particle radius $(R / r)$ and the applied particle forces $(F)$. Our model was checked to completely match with full axisymmetric computational fluid dynamics simulations (CFD), carried out in COMSOL MULTIPHYSICS. ${ }^{8}$

Figure 2(a) shows the 1D velocity profile of two separate particles (thin lines) with velocity $\left(V_{P}\right)$. If we initially focus on one particle and ignore boundary effects of channel walls, the velocity of the propelled fluid $\left(V_{F, P}\right)$ beside the particle is roughly inversely proportional to the distance $(z)$ from the particle center (in case $z>\sqrt{3}$ ), as confirmed analytically by the following derivation: ${ }^{7} \quad V_{F, P}=V_{P}-\frac{1}{2} V_{P}\left[(r / z)^{3}-3(r / z)^{1}\right.$ $+2]$. The total channel axis velocity profile is computed by a superposition of the two single particle profiles: ${ }^{7} V_{F, \text { axis }}$ $=V_{F, P(1)}+V_{F, P(2)}$. Thereby, we obtain a symmetric curve (bold line) where both particles experience an equal velocity increase by their hydrodynamic interactions: the particles will not move with respect to each other. The velocity enhancement is a function of the particles interspacing and reaches a maximum of $\sim 1.55$ times the single particle velocity at the point of touch. ${ }^{7}$ In case of a channel wall at distance $(R)$, similar curves are computed for single and inter-
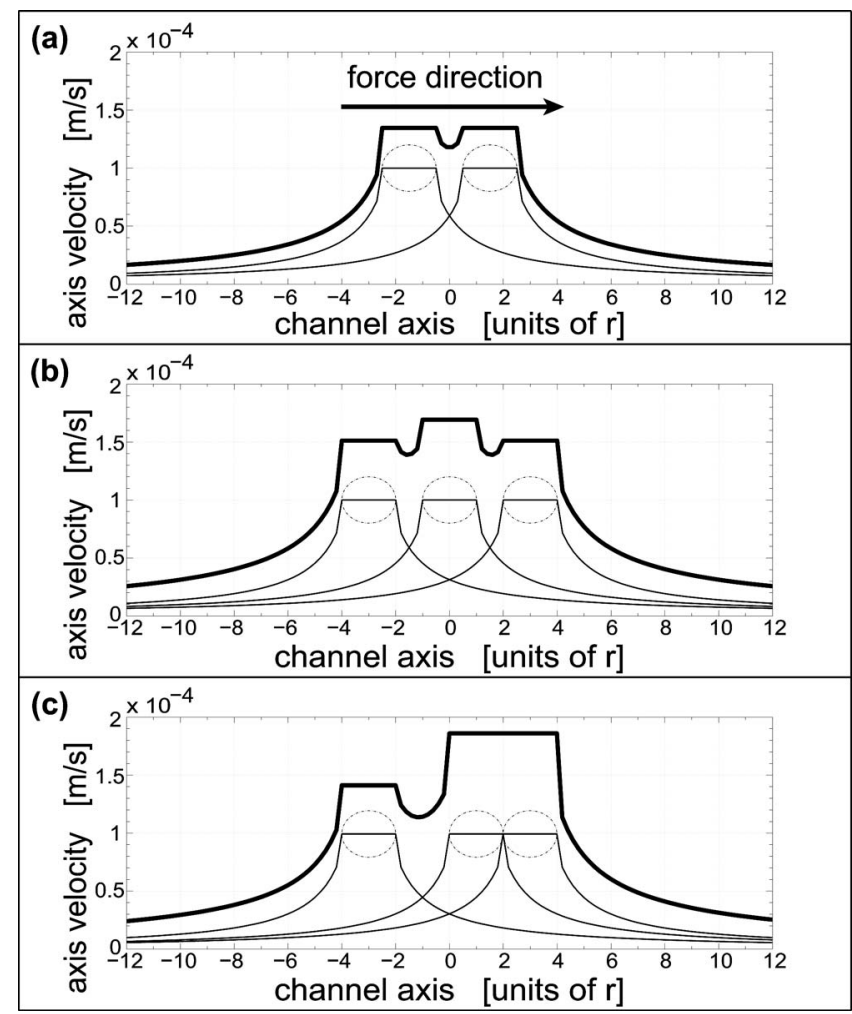

FIG. 2. (a) Calculated velocity profiles of two particles (thin lines), showing that the fluid velocity decrease is inversely proportional to the distance $(z)$ from the particle. The resulting axis velocity profile (bold line) is obtained by superposition of the two velocity curves, which demonstrates an even increase of both particle velocities: a constant spacing over time. (b) Hydrodynamic interactions of three particles give a higher velocity enhancement for the center particle by contributions of both neighbors. (c) The difference in velocities leads to formation of a twin that increases velocity with respect to the left particle and travels away.

acting particles; however, the power of the two $(r / z)$ ratio terms will both change and result in amplified particleparticle interactions (as long $R \gtrsim 2 r$ ).

In Fig. 2(b), a third particle is added. Superposition of the single particle profiles reveals that the middle particle reaches a higher velocity than the outer particles as a result of contributions from both neighbors. Over time, the middle particle will therefore approach the heading particle until a stable twin is formed, as shown in Fig. 2(c). As a result of the increased velocity for a twin, it will subsequently travel away from the left particle.

The observed twin intraspacing in case of magnetic driving forces is modeled using a dipole interaction approximation. ${ }^{4}$ We calculated that the dipolar repelling force $\left(\propto 1 / s^{4}\right)$ and the hydrodynamic twinning force $(\propto 1 / s)$ equilibrate at a twin intraspacing $(s)$ in the range of a particle diameter, confirmed by our observations shown in Fig. 1.

The described mechanisms also occur on a longer row of (n) particles that is modeled by $V_{\text {axis }}=V_{F, P(1)}+V_{F, P(2)}+\ldots$ $+V_{F, P(n)}$, where a released twin successively creates space for the subsequent particles to twin as observed in the experiments. The resulting polytwin system is able to regulate and stabilize its interspacing: a twin that approaches a second twin in front will enlarge its intraspacing and thereby reduce velocity. Conversely, the twin in the leading position will reduce its intraspacing (if present) and thereby accelerate to travel away from the following twin. If a twin catches up with a single particle, it almost collides but increases its in- 


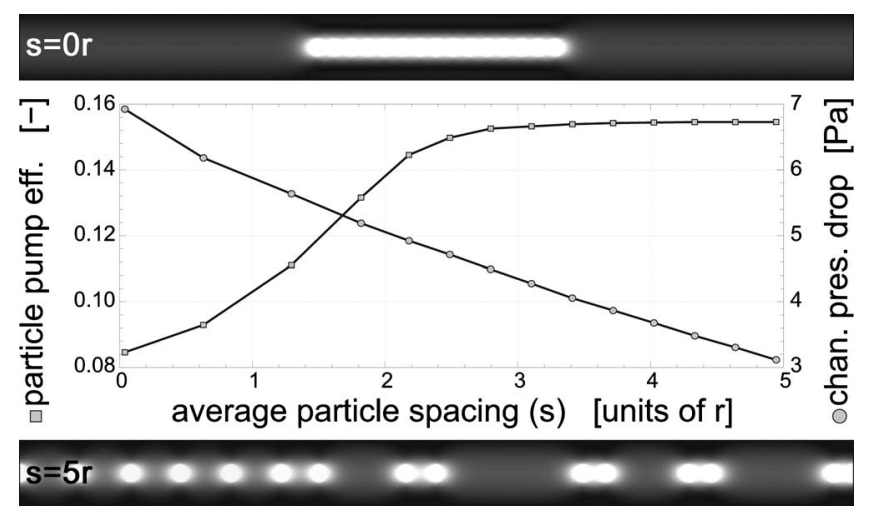

FIG. 3. Simulation of 15 particles $(r=10 \mu \mathrm{m})$ in a microchannel $(R$ $=50 \mu \mathrm{m}, L=1 \mathrm{~mm}$ ), starting as a full contact chain (top) and develops into a polytwin system (bottom). The pump efficiency per particle (ratio between fluid outflow velocity and average particle velocity) is given as a function of average particle spacing, and increases as twins are formed. Moreover, the induced pressure drop $\left(P_{\max }-P_{\min }\right)$ over the channel reduces by the increasing particle spacing.

traspacing to release the twin rear particle; subsequently, the leading particle forms a stable twin again with the initially single particle. ${ }^{8}$

The required time to develop a full polytwin system and the resulting twin interspacing can be optimized by tuning the initial particle configuration, the ratio of channel to particle radius $(R / r)$ and the applied particle forces $(F)$. The formation of twins may be disturbed by inhomogeneous force fields and dispersion in particle sizes that give velocity variations, or by off-axis particle motion due to weak hydrodynamic focusing (e.g., high $R / r$ ratio). System optimization and stability studies will therefore be the next points of interest in our investigations.

In view of the application of particles as integrated fluid drivers, the influence of the twin formation on the average fluid drag per particle (pump efficiency) and the channel pressure drop has been evaluated by full CFD simulations with similar conditions as the magnetic particle experiment. As twins form and get released from a long row of particles the average particle spacing increases, as shown in Fig. 3. This leads to a lower average particle velocity but enhances the particle pump efficiency, calculated by the ratio of fluid outflow velocity to average particle velocity. Furthermore, the spread out of fluid driving particles (or in this case, distant twins) in a long and narrow channel reduces local peak pressures to allow higher flow rates and minimize fluid ve- locity variations, which become increasingly important when downscaling fluidic systems.

To conclude, in magnetic and gravitation driven experiments we observed a hydrodynamic particle interaction effect that leads to successive formation of stable particle twins (in case of at least three particles) in low Reynolds number regimes. A linear 1D model based on the channel axis flow profile explains the self-organization by a superposition of particle velocity contributions that vary as a function of interspacing. The model is able to describe the reshuffling processes in a long row of particles, showing the successive twin formation and a regulation of inter- and intra-twin spacings, even in the presence of additional repelling forces. The microfluidic pump application based on actuated particles as fluid drivers profits from the twin self-regulation mechanisms by an enhanced pump efficiency per particle and a reduction in peak pressures over the microchannel. By tuning the direction and magnitude of the applied magnetic fields and channel to particle dimensions, the (long range) hydrodynamic- and (short range) magnetic particle interactions can be controlled to achieve optimum performance. Off-axis particle configurations and perpendicular alignments can also be formed this way and will be investigated in upcoming research. We believe that the presented experimental proof and theoretical understanding pave the way for actuated particles as well controlled integrated fluid drivers, particularly in miniaturized, high surface area, multiparticle/ multi channel systems.

${ }^{1}$ S. Haeberle and R. Zengerle, Lab Chip 7, 1094 (2007).

${ }^{2}$ M. W. J. Prins and M. Megens, Encyclopedia of Materials: Science and Technology, edited by K. H. J. Buschow, R. W. Cahn, M. C. Flemings, B. Ilschner, E. J. Kramer, and P. Mahajan Sand Veyssière (Elsevier, New York, 2007).

${ }^{3}$ R. J. S. Derks, A. Dietzel, R. Wimberger-Friedl, and M. W. J. Prins, Microfluid. Nanofluid. 3, 141 (2007).

${ }^{4}$ I. Petousis, F. G. A. Homburg, R. J. S. Derks, and A. Dietzel, Lab Chip 7, 1746 (2007).

${ }^{5}$ C. Mikkelsen, M. F. Hansen, and H. Bruus, J. Magn. Magn. Mater. 293, 578 (2005).

${ }^{6}$ J. J. Chalmers, Y. Zhao, M. Nakamura, K. Melnik, L. Lasky, L. Moore, and M. Zborowski, J. Magn. Magn. Mater. 194, 231 (1999).

${ }^{7}$ J. Happel and H. Brenner, Low Reynolds Number Hydrodynamics, 2nd ed. (Noordhoff, Leyden, 1973).

${ }^{8}$ See EPAPS Document No. E-APPLAB-92-080803 for a comparison movie between a full CFD simulation and the 1D model of 15 particles traveling in a microchannel. This document can be reached through a direct link in the online article's HTML reference section or via the EPAPS homepage (http://www.aip.org/pubservs/epaps.html). 